\title{
Um Estudo Sobre Pensamento Formal No Contexto dos Makerspaces Educacionais
}

\author{
Karen Selbach Borges (autora)', Léa da Cruz Fagundes (orientadora), Crediné \\ Silva de Menezes (coorientador) ${ }^{2}$
}

\author{
Instituto Federal de Educação, Ciência e Tecnologia do Rio Grande do Sul, \\ Campus Porto Alegre (IFRS) \\ Rua Cel. Vicente, 281 - Centro Histórico - Porto Alegre - RS - Brazil \\ Universidade Federal do Rio Grande do Sul (UFRGS) \\ Av. Paulo Gama s/n - Prédio 12201 - Porto Alegre - RS - Brazil \\ karen.borges@poa.ifrs.edu.br, \{leafagundes.credine\}gmail.com
}

\begin{abstract}
This research started from the hypothesis that digital manufacturing activities are able to lead the subject to perform complex mental operations, related to the formal operative level. From experiments conducted during Creativity Workshops at POALab FabLab, we found evidences that the use of digital fabrication machines, during the realization of the projects, enhanced the development of reflexive abstractions, the elaboration of hypotheses and the performance of second-order mental operations that are characteristic of formal thinking.
\end{abstract}

\begin{abstract}
Resumo. Este trabalho de pesquisa partiu da hipótese de que atividades de fabricação digital são capazes de levar o sujeito a realizar as operações mentais complexas, características do nível operatório formal. A partir de experimentos realizados durante as Oficinas de Criatividade no Contexto da Cultura Maker do POALab FabLab, obtivemos indicativos de que os projetos desenvolvidos, por utilizarem os equipamentos de fabricação digital, foram capazes de potencializar o desenvolvimento de abstraçôes reflexionantes, a elaboração de hipóteses e a realização de operações mentais de segunda ordem características do pensamento formal.
\end{abstract}

\section{Introdução}

Atualmente, inovação é sinônimo de prosperidade. O potencial de inovação de um país tem se mostrado como fator determinante para o crescimento da economia e, consequentemente, melhoria das condições de vida da população. Entretanto, a inovação depende tanto de pessoas quanto de recursos tecnológicos. De nada adiantam equipamentos sofisticados se não existem pessoas preparadas para usá-los e, principalmente, para criar soluções nas quais esses equipamentos possam ser empregados. Nesse sentido, a pesquisa de doutorado aqui relatada tinha como objetivo maior encontrar uma forma de capacitar as pessoas para se tornarem sujeitos produtores de inovações. Entendemos a inovação como um processo de criação a partir da conexão de diferentes saberes, com objetivo de produzir uma solução original e imbuída de valores (éticos e sociais), para um problema apresentado pela sociedade.

Sabe-se que a capacidade de criação de um sujeito é fruto da inteligência e de um processo de construção de conhecimento, temas estudados em profundidade por Jean Piaget. Segundo o pesquisador, a criação da novidade torna-se possível a partir do momento em que o sujeito atinge o nível operatório formal, entre 12 e 15 anos de idade. 
VIII Congresso Brasileiro de Informática na Educação (CBIE 2019)

Anais dos Workshops do VIII Congresso Brasileiro de Informática na Educação (WCBIE 2019)

Esse nível é caracterizado pelo raciocínio hipotético-dedutivo, que é o raciocínio que permite deduzir conclusões a partir de premissas em vez de deduzir diretamente a partir da realidade percebida. Ou seja, o sujeito é capaz de estabelecer hipóteses e, mentalmente, testá-las, descartá-las ou aceitá-las como soluções de problemas (PIAGET; INHELDER, 1978).

Entretanto, estudos mostram que nem todos os sujeitos adultos atingem o nível formal de desenvolvimento cognitivo. Cantelli, Borges e Mantovani de Assis (2002) concluíram que apenas 6,5\% dos sujeitos matriculados na modalidade EJA (Educação de Jovens e Adultos) encontravam-se no estágio operatório formal. Szymanski (2011), comprovou estatisticamente que a maioria dos sujeitos da pesquisa (alunos dos cursos de Atendente de Enfermagem), embora adultos cronologicamente, se encontravam no nível Operatório Concreto. Os resultados obtidos por estes pesquisadores brasileiros se repetem também em outros países. Pandey, Bhattacharya e Rai (1993) analisaram 884 estudantes indianos com idades entre 13 e 19 anos de idade, e concluíram que "uma grande porcentagem $(76.9 \%, 59.2 \%, 57.9 \%$ e $47.8 \%$ para as séries nona, décima, décima primeira e décima segunda) de estudantes estavam operando no nível formal)"(tradução nossa) (p. 428). No trabalho de Lis e Magro (1993) com 230 adolescentes italianos, os pesquisadores concluíram que "parte da nossa amostra não está operando no nível mais alto do pensamento formal" (tradução nossa) (p. 750).

A partir desses trabalhos, decidimos fazer uma investigação preliminar sobre o nível de desenvolvimento cognitivo dos alunos do curso em Tecnologias de Sistemas para Internet do Instituto Federal de Educação, Ciência e Tecnologia do Rio Grande do Sul (IFRS), campus Porto Alegre. Como resultado, verificamos que aproximadamente $15 \%$ dos estudantes operavam no nível concreto. Esse resultado nos levou a questionar: como querer que os jovens sejam sujeitos inovadores, se eles ainda nem conseguiram atingir o estágio de desenvolvimento cognitivo que proporcione isso? O que pode ser feito para alavancar a passagem para o operatório formal? Como aproveitar os recursos disponíveis dentro do IFRS-POA para dar suporte a esse processo?

Desde 2015 o IFRS-POA conta com um makerspace interligado a rede mundial de FabLabs: o POALab. Os makerspaces são, essencialmente, "espaços comunitário onde os membros compartilham acesso a ferramentas para produzir bens físicos [...] formulado em contraste com o termo hackerspaces, considerados mais focados em computadores e eletrônicos" (tradução nossa) (VAN HOLM, 2014). Já os FabLabs, são makerspaces organizados em rede, seguindo um modelo proposto pelo Centro de Bits e Átomos do MIT (Massachusetts Institute of Technology). O POALab, como atividade de extensão do IFRS câmpus Porto Alegre, tem como objetivo, não apenas oferecer acesso às tecnologias de fabricação digital mas, principalmente, servir como espaço interdisciplinar de aprendizagem, criatividade e inovação.

A aprendizagem através do fazer, da construção de artefatos físicos, está sustentada na pedagogia construcionista de Seymourt Papert, o qual observou que o desenvolvimento dos mecanismos cognitivos é privilegiado em situações em que o sujeito esteja conscientemente envolvido na construção de alguma coisa utilizando, para tanto, "objetos para pensar com". Com a evolução tecnológica esses objetos passaram da tartaruguinha do Logo a sofisticados kits de robótica, kits de automação (compostos por sensores, atuadores e controladores, como os Arduínos) e ferramentas de prototipação e fabricação digital, tais como fresadora, cortadora laser, plotter de recorte e impressora 3D.

A partir da disponibilidade dessas ferramentas no POALab e, considerando os resultados obtidos na investigação preliminar, chegamos a seguinte questão de pesquisa: como as atividades de fabricação digital podem apoiar o desenvolvimento do pensamento formal? Nossa hipótese era que atividades de fabricação digital seriam capazes de levar o sujeito a operar sobre abstrações, de desenvolver raciocínios hipotético-dedutivos e de realizar as operações mentais complexas, características do 
VIII Congresso Brasileiro de Informática na Educação (CBIE 2019)

Anais dos Workshops do VIII Congresso Brasileiro de Informática na Educação (WCBIE 2019)

nível operatório formal. Para avaliar esta hipótese foi necessário obter respostas às seguintes questões: Quando o pensamento formal é aplicado durante as atividades de fabricação digital?; As atividades de fabricação digital podem contribuir para o desenvolvimento do pensamento formal?; Qual seria uma abordagem pedagógica adequada para o uso do pensamento formal a partir do desenvolvimento de projetos de fabricação digital?

A investigação dessas questões foi conduzida dentro de um contexto de educação maker fundamentada em um recorte da epistemologia genética de Jean Piaget. A teoria dos estágios operatórios encontra-se descrita na seção 2 deste artigo. Na seção 3 apresentamos um levantamento sobre trabalhos também relacionados com o estudo do pensamento formal. Na seção 4 detalhamos a metodologia de pesquisa utilizada para a coleta e análise de dados, os quais são demonstrados e discutidos na seção 5. As respostas das questões de pesquisa são apresentadas na seção 6. As considerações finais (seção 7) e as referências encerram esse artigo.

\section{Fundamentação Teórica}

A pesquisa foi embasada nos estudos de Jean Piaget sobre os estágios de desenvolvimento cognitivo. Segundo o pesquisador, o desenvolvimento pode ser dividido em quatro períodos: sensório-motor: ocorre até os 2 anos de idade; préoperatório: ocorre na faixa dos 2 aos 7 anos de idade; operatório concreto: ocorre na faixa dos 7 aos 11 anos de idade; operatório formal: ocorre na faixa dos 12 aos 15 anos de idade. A passagem de um estádio para o outro não é condicionada à idade, mas sim ao desenvolvimento de esquemas que permitem o sujeito abstrair e realizar procedimentos ou operações sobre os objetos, modificando as suas estruturas cognitivas.

A partir dos 7 anos de idade, os esquemas mentais de uma criança passam a se organizar na forma de operações. "Uma operação é com efeito, uma ação efetiva ou interiorizada, tornado reversível e coordenada a outras operações, numa estrutura de conjunto que comporta leis de totalidade" (PIAGET, 1989 p. 111). A partir, então, do momento em que a criança torna-se capaz de reverter mentalmente uma ação, ela passa a ser considerada operatória.

No estágio operatório concreto as crianças necessitam do apoio de elementos concretos para desenvolver raciocínios. É o caso, por exemplo, das crianças que usam os dedos para realizar a operação matemática de adição. Já as crianças que se encontram no estágio formal conseguem desenvolver raciocínios puramente baseados em abstrações e, conforme a necessidade, representá-los através de formalismos como, por exemplo, equações, gráficos e esquemas.

A principal característica do estágio operatório-concreto é o surgimento das operações, sendo a reversibilidade (por inversão ou reciprocidade) a primeira delas. Além dessa, os sujeitos desse estágio de desenvolvimento também são capazes de realizar as operações de classificação (agrupamento de objetos conforme a semelhança de suas propriedades), seriação (ordenação de elementos considerando a ordem linear de grandeza desses elementos) e enumeração (seriação em função de suas posições no espaço ou tempo); operações espaciais (ocorrem com base em proximidades e distanciamentos), sobre estruturas aditivas ou multiplicativas (como as matrizes) e operações envolvendo tempo e velocidade. (PIAGET, 1973 e 1978) Todas essas operações são reversíveis, ou seja, podem ser desfeitas ou aplicadas em sentido contrário como, por exemplo, seriação na ordem inversa. Neste estágio, desenvolve-se também a capacidade de realizar antecipações e retroações, e são dados os primeiros passos na elaboração de hipóteses.

Já a principal característica do estágio formal é o fato de que o sujeito adquire a capacidade de separar o conteúdo da forma e, através da abstração, retirar da realidade 
VIII Congresso Brasileiro de Informática na Educação (CBIE 2019)

Anais dos Workshops do VIII Congresso Brasileiro de Informática na Educação (WCBIE 2019)

as informações necessárias para determinar possibilidades (hipóteses) e deduzir delas as suas consequências. Assim, diz-se que o pensamento formal é essencialmente hipotético-dedutivo e apoia-se nas operações proposicionais e combinatórias.

A partir do momento em que o sujeito domina as relações de causa e efeito, compreendendo que uma causa implica certo efeito, ele torna-se capaz de realizar deduções e estabelecer hipóteses. Para tanto, ele realiza operações proposicionais que visam verificar a validade ou não das hipóteses elaboradas. Entretanto, a relação causa e efeito nem sempre é unívoca; um resultado pode ter diferentes causas, obtidas pela combinação de fatores ou ocasionada por eventos casuais. Disso surgem os esquemas que darão ao sujeito condições de realizar operações combinatórias.

Além da operações combinatórias, os sujeitos formais são capazes de realizar operações sobre operações, também chamadas de operações de segunda ordem, tais como as operações de probabilidade, correlação (interdependência de duas ou mais variáveis), compensação e proporcionalidade (compreensão das relações parte-todo, frações) e coordenação de dois sistemas de referência, relatividade dos movimentos e das velocidades, e coordenação de equilíbrio mecânico (INHELDER; PIAGET, 1976).

\section{Trabalhos Relacionados}

Em pesquisas a partir de bases de dados nacionais encontramos a tese de Lemos (2015), a qual destacou-se pelo perfil escolhido para os sujeitos de pesquisa: adultos e idosos. A pesquisa tinha por objetivo descrever características do estágio operatório formal nessa etapa do desenvolvimento humano. Outro trabalho interessante foi a dissertação de Marques (2014) que demonstrou certa relação com a pesquisa de doutorado realizada na medida em que buscava analisar a influência das tecnologias na promoção do pensamento abstrato. $\mathrm{O}$ percurso metodológico adotado se assemelhava nos seguintes pontos: os sujeitos foram estudados a partir do seu envolvimento em um projeto de aprendizagem; as atividades de aprendizagem foram orientadas por uma metodologia (ensino baseado na investigação); trabalharam com tecnologias digitais tais como sensores de temperatura, vento e umidade, sistemas de georeferenciamento, computadores conectados à internet e equipados com sistemas de aquisição e tratamento de dados. A partir da análise dos dados coletados, Marques (2014, p. iv) conclui "sem generalizar, que o uso das tecnologias, inseridas em situações de aprendizagem significativas, favorece o desenvolvimento do pensamento abstrato".

No cenário internacional, encontramos diversas publicações sobre aprendizagem em makerspaces sem, entretanto, um aprofundamento nas questões relacionadas com o desenvolvimento cognitivo. Martinez e Stager (2013) possuem uma das publicações mais populares sobre o assunto, abordando, entre outras coisas, um modelo de aprendizagem chamado Think, Make, Improve. Petrich, Wilkinson e Bevan (2011) relatam que evidências de aprendizagem podem ser observadas através do engajamento, da colaboração e da solidariedade das crianças durante as atividades, e nos aspectos de intencionalidade e inovação das soluções elaboradas. O artigo de Kaun (2012) destacase pela análise do impacto cognitivo de atividades maker com crianças de quarta-série, envolvidas em uma atividade sobre energia e circuitos elétricos. Os dados coletados pela autora demonstraram que as crianças foram capazes de elaborar inferências e avaliações críticas, identificar os elementos de um problema e de definir estratégias para a resolução de problemas.

A partir dessa pesquisa sobre trabalhos relacionados, constatamos que, até o presente momento, não se tem conhecimento de trabalhos que investigam $o$ pensamento formal a partir do uso de tecnologias de fabricação digital. Assim, o caráter inédito da pesquisa de doutorado que aqui apresentamos consiste na análise sobre o uso e desenvolvimento do pensamento formal durante a realização de projetos de fabricação digital orientados por uma arquitetura pedagógica para aprendizagem em makerspaces educacionais. 
VIII Congresso Brasileiro de Informática na Educação (CBIE 2019)

Anais dos Workshops do VIII Congresso Brasileiro de Informática na Educação (WCBIE 2019)

\section{Metodologia da Pesquisa}

Sabendo que o fenômeno que estava sendo investigado nesta pesquisa era o desenvolvimento cognitivo e que, para isso, seria necessário observar e refletir sobre o comportamento, o discurso (falado ou escrito) e a capacidade de criação dos sujeitos, fomos buscar, na obra de Piaget e seus colaboradores, por orientações sobre como proceder na realização dos experimentos. Encontramos em Inhelder, Bovet e Sinclair (1977) o Método de Exploração Crítica, sobre o qual realizamos algumas adaptações com o objetivo de adequá-lo aos objetivos da pesquisa.

A partir disso, utilizamos um método de pesquisa baseado em observação e análise qualitativa, variando as experimentações e as maneiras de coletar, transcrever, apresentar e analisar os dados, conforme a questão de pesquisa a qual se desejava obter respostas. Para Inhelder e seus colegas

é preciso ter presente que, se o que nos interessa é entender como os sujeitos vão mudando ao longo do seu desenvolvimento, podemos utilizar diferentes estratégias para estudá-los .. Um método não é bom nem mau em si. Ele não pode ser julgado senão em função dos problemas ao qual é chamado a resolver e que, por sua vez, são orientados por perspectivas epistemológicas mais ou menos explícitas (Inhelder, Sinclair e Bovet apud in DELVAL, 2002 p. 33)

Os experimentos foram realizados na forma de Oficinas de Criatividade dentro do POALab. Durante essas oficinas os sujeitos foram desafiados a fabricar um artefato utilizando, para tanto, ferramentas de fabricação digital. Essas oficinas constituíam atividades associadas ao Programa de Extensão POALab. Foram ofertadas em três edições (2016/1, 2016/2 e 2017/2). Cada oficina possuía temáticas e durações diferentes, conforme a questão de pesquisa que definia o objetivo do experimento (Quadro 1).

Quadro 1 - Dados gerais sobre as Oficinas de Criatividade

\begin{tabular}{|c|c|c|c|c|}
\hline Nome & $\begin{array}{l}\text { Número de } \\
\text { Participantes }\end{array}$ & $\begin{array}{c}\text { Carga } \\
\text { Horária }\end{array}$ & Temática & Questão de pesquisa \\
\hline Oficina 1 & 12 & 20 horas & Luminárias & \multirow{3}{*}{$\begin{array}{l}\text { 1)Quando o pensamento } \\
\text { formal é aplicado durante as } \\
\text { atividades de fabricação } \\
\text { digital? } \\
\text { 3)Qual seria uma abordagem } \\
\text { pedagógica adequada para o } \\
\text { uso do pensamento formal a } \\
\text { partir do desenvolvimento de } \\
\text { projetos de fabricação digital? }\end{array}$} \\
\hline Oficina 2 & 12 & 45 horas & $\begin{array}{l}\text { Jogos de } \\
\text { tabuleiro }\end{array}$ & \\
\hline & & & & \\
\hline Oficina 3 & 8 & 32 horas & Barco a vela & $\begin{array}{l}\text { 2) As atividades de fabricação } \\
\text { digital podem contribuir para } \\
\text { o desenvolvimento do } \\
\text { pensamento formal? }\end{array}$ \\
\hline
\end{tabular}

Todas as oficinas foram oferecidas gratuitamente, podendo participar sujeitos a partir de 15 anos de idade. As oficinas 1 e 2 eram abertas à comunidade, já a Oficina 3 foi oferecida apenas para alunos de $1^{\circ}$ semestre dos cursos técnico ou superior tecnológico do IFRS, câmpus Porto Alegre. Para fins de análise dos dados da Oficina 3, foram considerados os sujeitos situados como Operatório Concreto em pelo menos um dos Testes Operatórios Coletivos (LONGEOT, 1979) e que tivessem realizado todas as atividades propostas pela pesquisadora. 
VIII Congresso Brasileiro de Informática na Educação (CBIE 2019)

Anais dos Workshops do VIII Congresso Brasileiro de Informática na Educação (WCBIE 2019)

Esses testes eram aplicados antes do início das atividades. Eles permitiam situar os sujeitos de pesquisa entre os estágios de desenvolvimento Operatório Concreto e Operatório Formal, conforme a escala de desenvolvimento do pensamento lógico (EDPL) proposta por Longeot( 1979). Os testes avaliavam a capacidade dos sujeitos de operarem sobre proposições lógicas (TOFLP), probabilidades e proporcionalidades (TOFP), e combinatórias (TOFC). Os testes foram adaptados para o formato de formulários eletrônicos, e disponibilizados via Internet, através dos seguintes endereços:

- TOFP - https://goo.gl/forms/OQ5Xm6vZp69eWJU73

- TOFLP - https://goo.gl/forms/Ygp9peBoPv4nIWb53

- $\quad$ TOFC - https://goo.gl/forms/VpYrTkYmD5wkjDK13

Para a realização das atividades experimentais era necessária a adoção de uma prática pedagógica que não apenas orientasse a condução das atividades, mas que também levasse os sujeitos a usarem o pensamento formal. Assim, criamos a APAME (Arquitetura Pedagógica para Aprendizagem em Makerspaces Educacionais), "que propõe uma organização de trabalho, cujas atividades envolvem o uso de tecnologias digitais e são amparadas pela epistemologia de Jean Piaget" (BORGES; MENEZES, 2018 p. 459).

As atividades realizadas pelos sujeitos participantes da pesquisa, bem como seu histórico de aprendizagem, eram registradas na forma de portfólios, os quais foram elaborados a partir do uso da ferramenta de textos colaborativos da Google. Sobre aquilo que era registrado, eram realizados comentários e questionamentos, inspirados no Método Clínico de Piaget (DELVAL, 2002) sobre as experiências vividas em laboratório.

$\mathrm{Na}$ etapa de análise dos dados, buscamos nas gravações e nos portfólios, por indícios das seguintes características do pensamento formal: capacidade de abstração, especialmente a abstração reflexionante; raciocínios elaborados a partir do levantamento de possibilidades; raciocínios hipotético-dedutivos; operações sobre combinatórias e proporções.

\section{Resultados e Discussões}

Os dados colhidos durante as oficinas de criatividade trouxeram evidências de uso do pensamento formal durante o desenvolvimento dos projetos de fabricação digital, além de indícios de melhorias na capacidade dos sujeitos de raciocinarem sobre abstrações, de elaborar hipóteses e de realizar operações de segunda ordem como combinações, proporções e probabilidades. Essas melhorias são indicativas de progressos do pensamento concreto em direção ao pensamento formal.

\subsection{Evidências de uso do pensamento formal}

Quando o sujeito se empenha em fabricar alguma coisa ele, a todo momento, se defronta com diversas possibilidades, as quais exigem lógica e abstração para determinar as melhores escolhas. No caso da fabricação de uma luminária, os sujeitos, a partir de seus relatos, trouxeram evidências dos "possíveis e necessários" à execução de seus projetos. Segundo Piaget (1985) quando o sujeito torna-se capaz de raciocinar sobre possibilidades, principalmente aquelas elaboradas a partir de um raciocínio hipotéticodedutivo, e identificar "co-possíveis quaisquer em número ilimitado" (PIAGET, 1985 p. 130), ele chegou ao patamar III, ou seja, o nível Operatório Formal.

Como exemplo, tomamos o caso de Angelo que relatou em seu portfólio os problemas de design constatados a partir da criação de um protótipo em papel de uma luminária (Figura 1) 


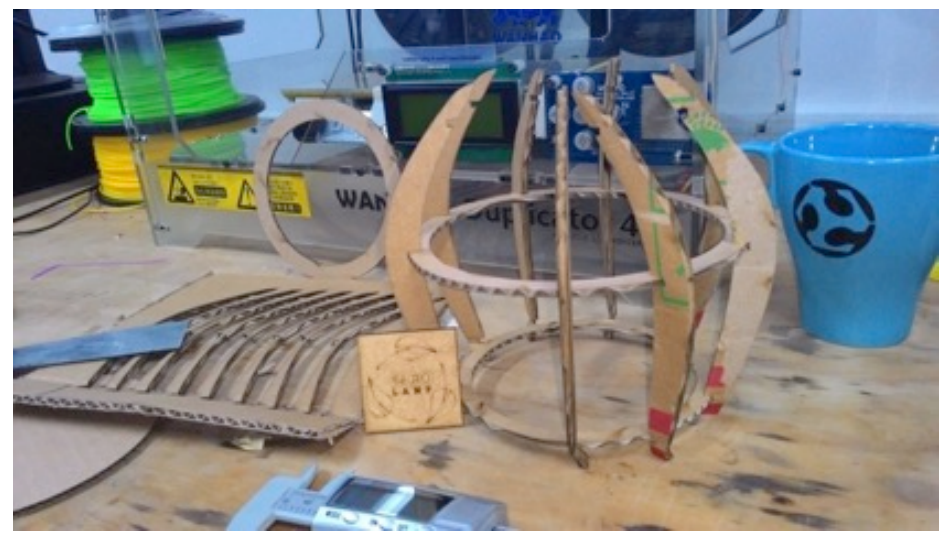

Figura 1 - Protótipo em papel da luminária decorativa extraído dos registros de Angelo

Sobre a possibilidade de correção dos problemas Angelo escreveu: "pensamos em ampliar a quantidade de hastes laterais de oito para doze e utilizar a mesma disposição e angulação utilizada para demarcar as horas em um relógio [...] pensamos em alterar também a base, estendendo o comprimento das hastes e removendo o circulo central e criando um suporte circular na base para encaixe e fixação das hastes". Observamos que Angelo escreve sobre duas possíveis formas de corrigir os problemas de design: aumentando a quantidade de hastes laterais e modificando a base de fixação das hastes. Essas são interpretadas como copossibilidades e, assim, caracterizam o pensamento formal.

\subsection{Indícios de desenvolvimento do pensamento formal}

Indícios de desenvolvimento do pensamento formal podem ser encontrados quando um sujeito situado na Escala do Pensamento Lógico como sendo Operatório Formal, demostra, por exemplo, ser capaz de definir combinações. Como exemplo, tomamos o caso de Júlia, que apresentara resultado Concreto no Testes Operatórios Coletivos relativo a lógica combinatória. Mostramos à ela a imagem da figura 2 e dissemos que essas foram as formas básicas usadas pelos participantes da oficina para construírem os seus barquinhos.

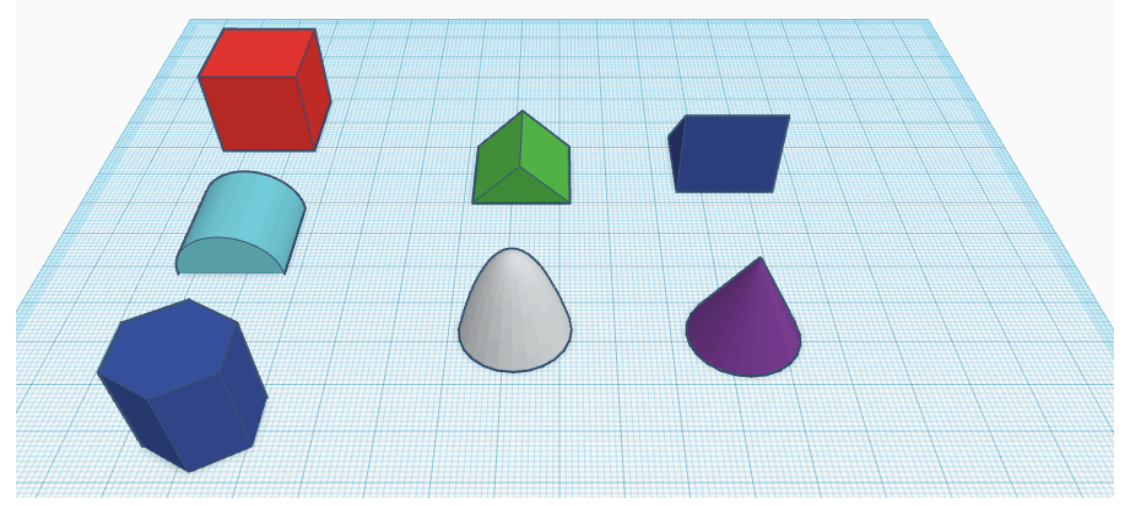

Figura 2 - Teste das combinações

Esclarecemos que na primeira coluna (da esquerda para direita) encontravam-se as três formas usadas para o corpo do barco; na segunda, as duas formas usadas para o bico do barco (a proa) e na terceira as duas formas usadas para a quilha. Dada essa explicação, perguntamos: Quantos modelos de barco diferentes são possíveis de serem fabricados, combinando essas formas? 
VIII Congresso Brasileiro de Informática na Educação (CBIE 2019)

Anais dos Workshops do VIII Congresso Brasileiro de Informática na Educação (WCBIE 2019)

Ela escreveu, então, as duas primeiras combinações possíveis a partir do cubo vermelho e, mesmo sem terminar de escrever as demais combinações, deu a resposta: 12. Para termos certeza do resultado do teste, seguimos perguntando: E se ao invés de duas opções para quilha, existissem três ? Julia pensou um pouco e respondeu: 18. Como se quisesse conferir o resultado ela fez a conta: 3 × 2 × $3=18$. Isso representa uma combinação de combinações, as quais são resultantes de uma operação de segunda potência, característica do estágio operatório formal.

\section{Conclusões}

Os dados obtidos com os experimentos realizados no POALab, serviram para elaborar as respostas às questões de pesquisa inicialmente colocadas.

\section{1 - Quando o pensamento formal é aplicado durante as atividades de fabricação digital?}

Quando criamos alguma coisa, consideramos as possibilidades sobre o design, sobre as ferramentas e os materiais que iremos utilizar, sobre os problemas que poderemos enfrentar, sobre como reduzir os custos do projeto, sobre a reação dos usuários, etc. Essa capacidade de antecipação é própria do pensamento formal e está relacionada com as condições do sujeito de operar sobre o real e o possível. Segundo Piaget (1985) “...o possível cognitivo é essencialmente invenção e criação" (p. 8).

Assim, durante um projeto de fabricação digital, podemos dizer que em toda a atividade que envolva a necessidade de antecipações, sejam elas materialmente ou estruturalmente possíveis, será necessário o uso do pensamento formal.

\section{2 - As atividades de fabricação digital podem contribuir para o desenvolvimento do pensamento formal?}

As atividades de fabricação digital que necessitam que o sujeito realize abstrações, construa e opere sobre hipóteses, e estabeleça relações de segunda ordem, como as correlações e compensações, podem contribuir com o desenvolvimento do pensamento formal. Observamos abstrações reflexionantes no uso de ferramentas de modelagem, as quais permitem que abstrações internas ao sujeito sejam transformadas em modelos com alto nível de complexidade, descritos na forma de desenhos bi ou tridimensionais; e nas atividades de configuração dos equipamentos, os quais demandam ajustes, físicos ou através do software de controle, que irão impactar diretamente no resultado do objeto que se pretende criar. Ou seja, através da atribuição de valores a uma série de variáveis, as propriedades de um objeto, até então imaginário, serão definidas.

Quando o sucesso da criação de um objeto depende de uma grande quantidade de variáveis é certo que o sujeito envolvido nessa atividade terá que, antecipadamente, elaborar um conjunto de hipóteses sobre os possíveis resultados a partir das diferentes configurações dessas variáveis. Nem todas as combinações fornecerão o resultado desejado, o que pode ser uma oportunidade de identificar as correlações a partir das quais são definidas as probabilidades de sucesso e fracasso.

\section{3 - Qual seria uma abordagem pedagógica adequada para o uso do pensamento formal a partir do desenvolvimento de projetos de fabricação digital?}

O pensamento formal, é fruto do surgimento de estruturas cognitiva que permitem ao sujeito operar sobre abstrações e hipóteses. Assim, as atividades realizadas durante o desenvolvimento de um projeto de fabricação digital devem ser orientadas no sentido de explorar possibilidades, porque essas são construídas sobre raciocínios hipotéticos, e de promover a reflexão, que é o componente principal do processo de abstração 
VIII Congresso Brasileiro de Informática na Educação (CBIE 2019)

Anais dos Workshops do VIII Congresso Brasileiro de Informática na Educação (WCBIE 2019)

reflexionante. A Arquitetura Pedagógica utilizada durante as Oficinas de Criatividade, conforme Borges e Menezes (2018), mostrou atender esses requisitos.

\section{Considerações Finais}

Os makerspaces constituem importantes ambientes de aprendizagem. A aparente desorganização desses espaços, sempre cheios de ferramentas, materiais alternativos para fabricação, protótipos em teste e elementos criativos, tornam os makerspaces um espaço constante de desequilibração. Os sujeitos, quando expostos à esse tipo de ambiente, envolvidos em projetos interdisciplinares, muitas vezes com áreas que não são de seu domínio, tem grandes chances de desenvolverem novos esquemas cognitivos ou, no mínimo, de melhorarem o seus esquemas conceituais pelo processo de autoregulação.

As atividades desenvolvidas durante as oficinas de criatividade nos mostraram que a aprendizagem em makerspaces se dá principalmente pela interação do sujeito com o objeto que está sendo criado e com as tecnologias digitais utilizadas em sua fabricação. Seja qual for o objeto a ser fabricado, observamos que as exigências de forma e funcionalidade impostas pelo próprio objeto são as primeiras resistências enfrentadas pelo sujeito que, ao superá-las, transforma o objeto, melhorando o seu design, e a si mesmo, na medida em que a compreensão do objeto modifica o seu sistema conceitual. Em seguida, e até mesmo concomitantemente, o sujeito se vê desafiado pelos softwares de modelagem e pelas máquinas de fabricação digital que, através das suas múltiplas possibilidades de configuração, tornam possível a concretização daquilo que antes só existia no plano abstrato.

O estudo do uso do pensamento formal no desenvolvimento de projetos envolvendo o uso de máquinas de fabricação digital para a produção criativa de artefatos é um dos diferenciais dessa pesquisa. Sabe-se, agora, que as atividades realizadas em makerspaces educacionais podem ajudar a desenvolver mecanismos cognitivos importantes, o que expande as possibilidades de aplicação da educação maker para além do ensino de conteúdos como Ciências, Tecnologias, Engenharia e Matemática (STEM). Além disso a Arquitetura Pedagógica para Aprendizagem em Makerspaces Educacionais, elaborada para nortear as ações realizadas durante as Oficinas de Criatividade, poderá servir, futuramente, para orientar propostas de atividades de aprendizagem nesses espaços de criatividade, prototipação e inovação.

Os desdobramentos dessa pesquisa podem seguir por diferentes caminhos. Registramos, como sugestão de continuidade dos estudos realizados, duas possíveis investigações: a primeira delas voltada para a parcela da população (jovem ou adulta) que apresenta atraso cognitivo. Nesse caso, o objetivo da pesquisa seria a definição de um conjunto de atividades criativas envolvendo fabricação digital, que pudesse ser usada para auxiliar o desenvolvimento desses sujeitos. A segunda sugestão de pesquisa seria a investigação do uso de atividades de fabricação digital como forma de minimizar os impactos da maturidade sobre a capacidade cognitiva de adultos e idosos. Outras possibilidades pesquisa se revelarão, também, em função do aumento do interesse pela área da neurociência, que pode oferecer um novo entendimento sobre as questões relacionados ao desenvolvimento cognitivo humano.

\section{Referências}

BORGES, K. S; MENEZES, C. S. Uma Arquitetura Pedagógica para Aprendizagem baseada na Fabricação Digital. In: VII Congresso Brasileiro de Informática na Educação - XXIX Simpósio Brasileiro de Informática na Educação Anais ..., Fortaleza, 2018. p. 457-465. Disponível em http://www.br-ie.org/pub/index.php/sbie/article/view/8002. Acesso em julho de 2019.

CANTELLI, V. C. B.; BORGES, R. R.; ASSIS, O. Z. M. DE. Avaliação do Desenvolvimento Intelectual de Alunos da Educação de Jovens e Adultos Braslleiros Numa Perspectiva 
VIII Congresso Brasileiro de Informática na Educação (CBIE 2019)

Anais dos Workshops do VIII Congresso Brasileiro de Informática na Educação (WCBIE 2019)

Piagetiana. In: VIII Congresso GalaicoPortuguês de PsicoPedagogia. Universidade Minho. Anais..., Portugal, 2005. p. 863 - 878 Disponível em http://www.educacion.udc.es/grupos/gipdae/documentos/ congreso/viiicongreso/pdfs/96.pdf. Acesso em maio de 2018.

DELVAL, J. Introdução à Prática do Método Clínico: descobrindo o pensamento das crianças. Porto Alegre: Artmed, 2002.

INHELDER, B.; BOVET, M.; SINCLAIR, H. Aprendizagem e Estruturas do Conhecimento. São Paulo: Saraiva, 1977.

INHELDER, B.; PIAGET, J. Da Lógica da Criança à Lógica do Adolescente: ensaio sobre a construção das estruturas operatórias formais. São Paulo: Pioneira, 1976.

KAUN, K. P. A Cognitive Analysis of the Benefits of Maker Kids. 2012. Disponível em https://www.changemakers.com/sites/default/files/competition_entry_form_files/cognitive _analysis_of_the_benefits_of_maker_kids.pdf. Acesso em setembro de 2019.

LANCI. S. et. al. Developing a Measure of Engineering Students' Makerspace Learning, Perceptions, and Interactions. American Society of Engineering Education, v. 22089, p. 1-12. Disponível em http://par.nsf.gov/biblio/10087318. Acesso em abril de 2019.

LEMOS, M. F. L., Características Procedimentais de Adultos e Idosos que Atingiram Nível de Desenvolvimento Operatório Formal em Provas da Escala de Desenvolvimento do Pensamento Lógico (EDPL) e no Jogo Quarto, Tese (Doutorado em Psicologia), Universidade Federal do Espírito Santo, Vitória(ES), 2015. Disponível em $\mathrm{http} / / /$ repositorio.ufes.br/handle/10/9090. Acesso em julho de 2019.

LIS, A.; MAGRO, T. Study of Longeot's test of formal operational thinking in a group of Italian adolescents. Perceptual and motor skills, v. 76, n. 3, p. 739-752, 1993. Disponível em https://doi.org/10.2466/pms.1993.76.3.739. Acesso em julho de 2019.

LONGEOT, F. L'Echelle de développement de la Pensée Logique. Issy-les-Moulineaux, EAP, 1979.

MARQUES, I.C.G.M. A influência das tecnologias na promoção do pensamento abstrato: um estudo de caso no $1^{\circ}$ ciclo do ensino básico. Dissertação (Mestrado em Educação e Multimidia). Instituto Politécnico de Viseu, Portugal. Disponível em http://hdl.handle.net/10400.19/2963. Acesso em julho de 2019.

MARTINEZ, S. L.; STAGER, G. Invent to Learn: Making, Thinkering and Engineering in the Classroom. Torrance: Constructing Modern Knowledge Press, 2013.

PANDEY, N. N.; S.B.BHATTACHARYA; RAI, V. K. Longeot test of cognitive development in Indian context. Studies in Educational Evaluation, v. 19, p. 425-430, 1993. Disponível em https://doi.org/10.1016/S0191-491X(10)80007-0. Acesso em julho de 2019.

PETRICH, M.; WILKINSON, K.; BEVAN, B. It Looks Like Fun, But Are They Learning ? In: HONEY, M.; KANTER, D. E. (Eds.). Design, Make, Play: Growing the Next Generation of STEM Innovators. Routledge, 2013. p. 50-70.

PIAGET, J. A Epistemologia Genética. 2. ed. Rio de Janeiro: Vozes, 1973.

PIAGET, J.; INHELDER, B. A Psicologia da Criança. Rio de Janeiro: Difel, 1978.

PIAGET, J. O possível e o necessário: evolução dos possíveis na criança. Vol.1. Porto Alegre: Artes Médicas, 1985.

PIAGET, J. Seis Estudos de Psicologia. 24. ed. Rio de Janeiro: Forense Universitária, 1989.

SZYMANSKI, M. L. S. A difícil aprendizagem de tarefas que exigem um raciocínio complexo. Boletim Técnico do Senac, v. 37, n. 1, p. 24-33, 19 abr. 2011. Disponível em http://www.bts.senac.br/index.php/bts/article/view/199. Acesso em julho de 2019.

VAN HOLM, E.J. What are Makerspaces, Hackerspaces, and Fab Labs? Disponível em http://ssrn.com/abstract=2548211. Acesso em setembro de 2019. 